\title{
URV ESPRIT for Tracking Time-Varying Signals
}

\author{
K. J. Ray Liu, Senior Member, IEEE, Dianne P. O'Leary, G. W. Stewart, and Yuan-Jye J. Wu
}

\begin{abstract}
ESPRIT is an algorithm for determining the fixed directions of arrival of a set of narrowband signals at an array of sensors. Unfortunately, its computational burden makes it unsuitable for real time processing of signals with time-varying directions of arrival. In this work we develop a new implementation of ESPRIT that has potential for real time processing. It is based on a rank-revealing URV decomposition, rather than the eigendecomposition or singular value decomposition used in previous ESPRIT algorithms. We demonstrate its performance on simulated data representing both constant and time-varying signals. We find that the URV-based ESPRIT algorithm is effective for estimating time-varying directions-of-arrival at considerable computational savings over the SVD-based algorithm.
\end{abstract}

\section{INTRODUCTION}

$\mathbf{E}$ SPRIT [1] is a method for determining directions-ofarrival (DOA) of a set of narrowband signals impinging on an array of $m$ sensors. It handles array geometries almost as general as those of the MUSIC algorithm [2] at a significant computational savings.

A key limitation of both the MUSIC and ESPRIT algorithms is the work required to process a new sample. At the heart of the algorithms is the separation of the $m$ dimensional sample space into an approximate signal subspace and an approximate noise subspace. Usually this separation is done by computing the eigendecomposition of the estimated covariance matrix, or part of the singular value decomposition of the data matrix. Unfortunately, these decompositions require $O\left(\mathrm{~m}^{3}\right)$ operations to update, making the algorithms unsuitable for real-time computation. Some attempts have been made to reduce the updating complexity by maintaining an approximate singular value decomposition (e.g., [3], [4]), but we believe that better results can be obtained using an alternate decomposition.

Recently, Stewart [5] has introduced the rank-revealing URV decomposition, a new matrix decomposition that produces the signal and noise subspaces, but can be updated in $O\left(\mathrm{~m}^{2}\right)$ time sequentially and in $O(\mathrm{~m})$ time on an array of $m$ processors. This means that algorithms that previously

Manuscript received October 21, 1992; revised April 8, 1994. This work was supported by: ONR Grant N00014-93-1-0566; NSF Grant MIP-9309506; the Institute for Mathematics and Its Applications at the University of Minnesota, AFOSR Grant 87-0158; the Graduate School General Research Board of the University of Maryland; and AFOSR Grant 87-0158. The associate editor coordinating the review of this paper and approving it for publication was Prof. Daniel Fuhrmann.

K. J. R. Liu is with the Department of Electrical Engineering and Institute of Systems Research, University of Maryland, College Park, MD 20742 USA.

D. P. O'Leary and G. W Stewart are with the Department of Computer Science and Institute for Advanced Computer Studies, University of Maryland, College Park, MD 20742 USA.

Y.-J. J. Wu is with the Applied Mathematics Program, University of Maryland, College Park, MD 20742 USA.

IEEE Log Number 9406037. depended on eigendecomposition or singular value decomposition may now be practical in real time applications, provided the URV decomposition can be successfully substituted. Boman, Griffin, and Stewart [6] have already exploited this fact to accelerate the MUSIC algorithm. The purpose of this paper is to investigate the use of the URV decomposition in timevarying signal processing using ESPRIT.

\section{THE ESPRIT ALGORITHM}

Roy and Kailath [1] noted that the ESPRIT idea is independent of the choice of matrix decompositions used in its implementation. In this section we discuss alternatives for these decompositions.

\section{The Background}

We consider $d$ narrow-band plane waves simultaneously incident on a planar array of $m$ sensors ( $m$ even), arranged in $m / 2$ doublet pairs. The displacement between sensors in a pair is constant in both direction and magnitude $\Delta$, but the location of each pair is arbitrary. The wave sources are assumed to be located in the same plane, and the location of each source is specified by a single parameter $\theta_{i} \in[-\pi, \pi]$, the DOA of the $i$ th source. Quantities related to the first and second sensors in each pair are subscripted by 1 and 2, respectively. All vectors are column vectors.

Given data from the array of sensors, the DOA estimation problem is to locate the directions of the sources. If the narrowband signals have the same known center frequency $\omega_{0}$, then the DOA problem can be described by a simple model. The relationship between the unknown signal $s(t) \in \mathcal{C}^{d}$ and the sensor output $x_{1}(t) \in \mathcal{C}^{m / 2}$ and $x_{2}(t) \in \mathcal{C}^{m / 2}$ is given by

$$
\begin{aligned}
& x_{1}(t)=A s(t)+e_{1}(t) \\
& x_{2}(t)=A \Phi s(t)+e_{2}(t)
\end{aligned}
$$

or

$$
x(t)=\left(\begin{array}{c}
A \\
A \Phi
\end{array}\right) s(t)+e(t)
$$

where $e(t)$ is the measurement noise, and $A \in \mathcal{C}^{m / 2 \times d}$ is the unknown matrix of array responses or array steering vectors. The diagonal matrix $\Phi$ is also unknown, and is related to the phase delays between the sensors in each doublet pair:

$$
\phi_{i}=e^{j \omega_{0} \Delta \sin \theta_{i} / c}, \quad i=1, \ldots, d .
$$

Our task is to estimate the number of signals $d$ and the directions-of-arrival, $\theta_{i}$. For this it is sufficient to estimate the matrix $\Phi$, which is the idea underlying ESPRIT. 
Let the $n$ columns of

$$
X=\left(\begin{array}{l}
X_{1} \\
X_{2}
\end{array}\right)
$$

form an ensemble of $n$ snapshots, and let $S$ be the corresponding matrix of signals. For noise free signals, $X$ has rank $d$, and if the $d$ columns of

$$
\left(\begin{array}{l}
V_{1} \\
V_{2}
\end{array}\right)
$$

form a basis for the column space of $X$, it follows from (1) and (2) that

$$
\left(\begin{array}{l}
V_{1} \\
V_{2}
\end{array}\right)=\left(\begin{array}{c}
A S B \\
A \Phi S B
\end{array}\right)
$$

where $S B$ is a nonsingular matrix of order $d$.

Next let the rows of

$$
\left(W_{1} W_{2}\right)
$$

form a basis for the row space of $\left(V_{1} V_{2}\right)$. It follows from (7) that $W$ can be written in the form

$$
\left(W_{1} W_{2}\right)=(C A S B \quad C A \Phi S B)
$$

where $C A$ is a nonsingular matrix of order $d$. Since $C A$ and $S B$ are nonsingular and $\Phi$ is diagonal, the diagonal elements of $\Phi$ are the eigenvalues of the pencil

$$
W_{2}-\lambda W_{1}=(C A) \Phi(S B)-\lambda(C A)(S B) .
$$

Given $\Phi$, the directions of arrival can be found from (4).

The above description of ESPRIT leads to many algorithms, even in the absence of noise, since there are many choices of bases. For example, if the bases $\left(V_{1}^{\mathrm{H}} V_{2}^{\mathrm{H}}\right)^{\mathrm{H}}$ and $\left(W_{1} W_{2}\right)$ are chosen to be orthonormal, then the resulting pencil is orthogonally equivalent to the pencil resulting from using the singular value decomposition to implement total least squares ESPRIT (the variant studied in this paper). In the presence of noise, one is faced with the additional problem of estimating the subspaces and their dimensions. The role of specific decompositions in ESPRIT algorithms is to make these estimations possible.

Thus the computational burden in ESPRIT is in choosing the matrices $B$ and $C$ that define the required bases. In particular, in the absence of noise, virtually any full rank matrices $B$ and $C$ will do (technically, any full rank matrices except those in a set of measure zero). Although we do not pursue this approach here, we feel it has the potential to yield fast algorithms that will work when there is a good signal-to-noise ratio.

When noise is present, then no matter how the matrices $B$ and $C$ are chosen, the computed basis matrices are only approximations to the true bases. It is necessary to choose these two matrices in a way that controls the effects of the noise.

\section{SVD ESPRIT}

The SVD ESPRIT algorithm finds the required bases using the singular value decomposition. We determine unitary matrices $U$ and $V$ such that

$$
X^{\mathrm{H}}=U D V^{\mathrm{H}}
$$

where the diagonal elements of $D=\operatorname{diag}\left(\delta_{1}, \delta_{2}, \ldots, \delta_{m}\right)$ are nonnegative and in descending order of magnitude. If $\delta_{d}$ is judged to be sufficiently large and $\delta_{d+1}$ is judged to be sufficiently small, then we conclude that there are $d$ signals, and the first $d$ columns of $V$ furnish the required (approximate) basis for the signal subspace.

A basis for the row space of $\left(V_{1} V_{2}\right)$ can also be determined by calculating an SVD. Specifically, let

$$
\left(V_{1} V_{2}\right)=T D_{V} W^{\mathrm{H}}
$$

be the SVD of $\left(V_{1} V_{2}\right)$. Then the first $d$ rows of $W^{\mathrm{H}}$ form the required basis $\left(W_{1} W_{2}\right)$.

The matrices $V$ and $D$ can be computed directly from the original matrix $X$, and this is the best algorithm in applications with very small singular values and high accuracy demands. However, for the DOA problem there are less costly alternatives: from (11), it follows that

$$
X X^{\mathrm{H}}=V D^{2} V^{\mathrm{H}} .
$$

Thus $V$ is the matrix of eigenvectors of the Hermitian crossproduct matrix $X X^{\mathrm{H}}$. This computational approach is particularly attractive, because the cross-product matrix can be easily updated as signals arrive.

Even with these economies, the SVD ESPRIT algorithm is expensive, requiring the $O\left(\mathrm{~m}^{3}\right)$ solution of an eigenvalue problem with each snapshot. Unfortunately, updating the eigendecomposition results in another $O\left(\mathrm{~m}^{3}\right)$ algorithm, though the order constant is smaller [7]. Recently, techniques for approximately updating an eigendecomposition have been proposed [3], [4], and they show some promise. However, in this paper we consider an alternative decomposition that can be updated in $O\left(\mathrm{~m}^{2}\right)$ time.

\section{URV ESPRIT}

The rank-revealing URV decomposition expresses $X^{\mathrm{H}}$ in the form

$$
X^{\mathrm{H}}=U R V^{\mathrm{H}}=U\left(\begin{array}{cc}
\tilde{R} & F \\
0 & G
\end{array}\right) V^{\mathrm{H}}
$$

where the columns of $U$ and $V$ are orthonormal, $\tilde{R}$ and $G$ are upper triangular of orders $d$ and $m-d$, and $F$ and $G$ are small in norm. This decomposition reveals that $X$ is within $\sqrt{\|F\|^{2}+\|G\|^{2}}$ of the matrix $\hat{X}$ of rank $d$ obtained by setting $F$ and $G$ in (14) to zero. The $U$ and $V$ of the URV decomposition are different than those of the SVD, but still provide approximate bases (exact in the absence of noise) for the required spaces. The sine of the largest canonical angle between the SVD noise space and the URV noise space is 
bounded by

$$
\tau \equiv \frac{\|F\|_{2}}{\left\{1-\left(\frac{\|G\|_{2}}{\sigma_{\min }(\tilde{R})}\right)^{2}\right\} \sigma_{\min }(\tilde{R})}
$$

where $\sigma_{\min }$ denotes the smallest singular value and the quantity in parentheses in the denominator is assumed to be less than one [8]. Thus, although both bases are only approximations to the true basis, they are close to each other. The column space of $\hat{X}$ is the space spanned by the first $d$ columns of $V$, and those columns are therefore a natural candidate for the basis required by the ESPRIT algorithm. The matrix $U$ is unnecessary and is not stored or updated.

In the same way, the URV decomposition of the matrix $\left(V_{1} V_{2}\right)$ can be used to determine the matrices $W_{1}$ and $W_{2}$ of the ESPRIT algorithm. We call the resulting algorithm the URV ESPRIT algorithm.

The URV decomposition can be updated in $O\left(\mathrm{~m}^{2}\right)$ time (and in $O(m)$ time on a linear array of $m$ processors). The updating procedure consists of two parts: an incorporation step and a deflation step. The incorporation is analogous to the standard update of a QR decomposition [9]; however, special care is taken that only the first column of $F$ and $G$ increases in norm. This corresponds to the fact that the addition of a row to a matrix can increase its rank by at most one. After the update, a condition estimator [10] is used to test $\tilde{R}$ for rank degeneracy, and a deflation step reduces the norm of the last column of $\tilde{R}$. If a degeneracy is detected, a refinement step is performed to bring the decomposition closer to diagonal form. All transformations are accomplished by plane rotations, and the algorithm is stable. For complete details, see Section III.

\section{Determining the Number of Signals}

In the time dependent problem, the sensors receive a new data sample at each time unit. We consider two common approaches to discounting the old data in order to develop reliable estimates of the current DOA's.

The first is rectangular windowing. In this method only the most recent $n$ data samples are retained, the earlier ones being discarded as irrelevant. Thus, at time $N$, we work with the data samples

$$
X=\left(x_{N-n+1}, \ldots, x_{N-1}, x_{N}\right) .
$$

This approach requires that we be able to downdate as well as update our decompositions.

The second approach is exponential windowing, which uses forgetting factors to discount the data in a more gradual way. As each new data sample is received, all old data is multiplied by a number $\mu$ between 0 and 1 , so that at time $N$ we work with the data samples

$$
X=\left(\mu^{N-1} x_{1}, \ldots, \mu x_{N-1}, x_{N}\right) .
$$

For exponential windowing, rounding error does not accumulate in the $R$ factor [11], [12]. Moreover, provided that orthogonality is maintained, neither does it accumulate in the $V$ factor [13]-[15].
Recent results on QR and URV downdating using the Linpack algorithm [16] or Chamber's algorithm [17] have shown that a sequence of updates and downdates can be quite stable [18]. Provided the sequence can be carried to completion, any well-conditioned leading principal submatrix of the final $R$ factor will be computed accurately. Ironically, computational difficulty arises only in low-noise problems: relative to $\|R\|$ the noise level should be above $\sqrt{n(m-d)}$ times the rounding unit; otherwise the downdate can fail to exist.

Since the above analysis considers only one-sided orthogonal transformations of the data, it does not, strictly speaking, apply to the URV algorithm. However, the modifications are obvious, and the results of our simulations confirm the stability.

The two forms of windowing represent two distinct algorithms, even when rounding error does not enter the picture. In evolving systems they will cause different numbers to be computed, and the errors in those quantities will have different statistical properties. Since neither can be excluded on grounds of numerical instability, it is desirable to place them side by side and see how they behave.

For the SVD, the sum $\delta_{d+1}^{2}+\cdots+\delta_{m}^{2}$ approximates the sum of squares of the projection of the error onto the orthogonal complement of the signal subspace (i.e., onto the noise subspace). If the individual components of the noise have (known) variance $\sigma^{2}$, then the statistical properties of the sum are well-understood. Its expected value is $n(m-d) \sigma^{2}$ for rectangular windowing and approximately $(m-d) \sigma^{2} /\left(1-\mu^{2}\right)$ for exponential windowing. Consequently, it is reasonable to choose the smallest $d$ satisfying

$$
\begin{aligned}
& \sqrt{\delta_{d+1}^{2}+\cdots \delta_{m}^{2}} \\
& \leq\left\{\begin{aligned}
\psi_{d} \sigma \sqrt{n(m-d)} & \text { for rectangular windowing, } \\
\psi_{d} \sigma \sqrt{\frac{m-d}{1-\mu^{2}}} & \text { for exponential windowing. }
\end{aligned}\right.
\end{aligned}
$$

Here $\psi_{d}>1$ is a factor chosen to make it unlikely that the dimension of the signal subspace is overestimated.

The URV quantity $\|G\|$ is the analogue of $\sqrt{\delta_{d+1}^{2}+\cdots+\delta_{m}^{2}}$ for the SVD. Consequently we choose the smallest $d$ satisfying

$$
\|G\| \leq \begin{cases}\psi_{d} \sigma \sqrt{n(m-d)} & \text { for rectangular windowing, } \\ \psi_{d} \sigma \sqrt{\frac{m-d}{1-\mu^{2}}} & \text { for exponential windowing. }\end{cases}
$$

These criteria are applied at the deflation step.

However, during the incorporation step a decision must be made as to whether $G$ has grown in norm due to an increase in rank. Here we use the same criterion, but with a different factor $\psi_{u}$ replacing $\psi_{d}$. As $\psi_{u}$ grows, the signal subspace changes less frequently. Thus, $\psi_{u}$ can be seen as a factor that controls the accuracy of the approximate signal subspace. In applications where only low accuracy is required, it may be taken large with a resulting savings in work. An alternative of this kind is not available for the SVD.

The advantage of our computational procedure is speed and simplicity, important features when tracking time-varying signals. Our simulations will show that such a scheme performs fairly well under a reasonable signal-to-noise ratio. If 
the variance of the noise is not known, or if the signals are moving rapidly, then the above procedure is no longer justified. Alternate schemes such as the well-known Akaike information criterion (AIC) and minimum description length (MDL) approaches [19] can be incorporated to obtain a better estimate of $d$ at the expense of additional computations. The use of parametric methods [20] can provide even better performance.

The AIC and MDL approaches both involve minimizing, with respect to $k$, a function of the sum and the product of the $k$ smallest eigenvalues of the sample covariance matrix $X X^{\mathrm{H}}$. Suppose that in (14) we have partitioned so that $G$ has dimension $k$. Denote the smallest eigenvalues of $X X^{\mathrm{H}}$ by $\lambda_{i}$, $i=1, \ldots, k$, and denote the eigenvalues of $G^{\mathrm{H}} G$ by $\hat{\lambda}_{i}$. Then Mathias and Stewart [8] have shown that

$$
\lambda_{i}=\left(1-\tau_{i}\right) \hat{\lambda}_{i}
$$

where

$$
0 \leq \tau_{i} \leq \frac{\|F\|_{2}}{\sigma_{\min }(\tilde{R})} \tau \equiv \hat{\tau}
$$

where $\tau$ is defined in (15). Thus, the product of the $k$ eigenvalues satisfies

$$
(1-\hat{\tau})^{k} \leq \frac{\prod \lambda_{i}}{\prod \hat{\lambda}} \leq 1 .
$$

Similarly, the sum of the eigenvalues satisfies

$$
\left|\sum \lambda_{i}-\sum \hat{\lambda}_{i}\right| \leq k \hat{\tau} \hat{\lambda}_{k}
$$

Thus, the accuracy with which we approximate the sum and product depends on the effort expended in keeping $F$ small in norm, and this can be adjusted as necessary. The sum and product of the eigenvalues of $G^{\mathrm{H}} G$ is, of course, available without computing the eigenvalues: we only require the sum of the main diagonal elements of $G^{\mathrm{H}} G$ and the square of the absolute value of the determinant of the triangular matrix $G$.

It is important to note that even though each of these methods can be easily applied using the URV, none of them takes account of the movement of the signals, and thus none can be justified theoretically for tracking of nonstationary signals.

\section{THE COMPUTATIONAL ALGORITHMS}

In this section we give a more detailed specification of the algorithms we use.

\section{The Time-Varying URV ESPRIT Algorithm}

Suppose that we already have a rank-revealing URV decomposition of the data matrix from the previous time. For rectangular windowing, we also save the most recent $n$ data samples. One step of the URV ESPRIT algorithm is defined as:

a) Obtain the new data sample $x$.

b) Update the previous rank revealing URV decomposition of the matrix of data samples $\left(X^{\mathrm{H}}\right)$ by downdating and updating the previous factors if rectangular windowing is used, or updating the previous factors if exponential windowing is used. c) Estimate the number of sources $d$ (i.e., the rank of $X$ ) using a tolerance of $\psi_{d}$ times the expected value of the noise. (The parameter $\psi_{d}$ is chosen by the user.)

d) The basis for the signal subspace (the range of $X$ ) is $E_{X}$, equal to the first $d$ columns of the $V$ factor in the URV decomposition.

e) Partition $E_{X}$ into $m / 2 \times d$ blocks corresponding to the two sets of sensors:

$$
E_{X}=\left(\begin{array}{l}
E_{1} \\
E_{2}
\end{array}\right)
$$

and compute a rank-revealing URV decomposition of $\left(E_{1} E_{2}\right)$ :

$$
\left(E_{1} E_{2}\right)=W \Psi V^{\mathrm{H}} .
$$

f) Partition $V$ into $d \times d$ blocks as

$$
V=\left(\begin{array}{ll}
V_{11} & V_{12} \\
V_{21} & V_{22}
\end{array}\right)
$$

and calculate the eigenvalues $\phi_{i}$ of $-V_{12} V_{22}^{-1}$ (or, equivalently, the eigenvalues of $V_{11}^{-H} V_{21}^{\mathrm{H}}$ ).

g) Estimate the DOS's from $\phi_{i}$ using (4).

\section{The URV Decomposition Algorithm}

Suppose $A$ is the current matrix, factored as $U R V^{\mathrm{H}}$, where $U$ is unitary (and neither stored nor updated),

$R$ is right triangular,

$V$ is unitary, and

$d$ is the estimated rank of $A$.

(Initially, set $R$ to a square matrix of zeros, $d$ to zero, and $V$ to the identity matrix.) $R$ is maintained with a graded structure, so that the column norms tend to form a decreasing sequence. We denote the incoming row by $z^{H}$, and the outgoing one (if there is one) by $y^{H}$.

Assuming that the standard deviation $\sigma$ of the noise is known, we assign two tolerances. We set the tolerance for increasing the estimate of the rank of $A$ to

$$
\text { tol_u }= \begin{cases}\psi_{u} \sigma \sqrt{n(m-d)} & \text { for rectangular windowing, } \\ \psi_{u} \sigma \sqrt{\frac{m-t}{1-\mu^{2}}} & \text { for exponential windowing. }\end{cases}
$$

The tolerance for decreasing the estimate is

tol_d $= \begin{cases}\psi_{d} \sigma \sqrt{n(m-d+1)} & \text { for rectangular windowing, } \\ \psi_{d} \sigma \sqrt{\frac{m-d+1}{1-\mu^{2}}} & \text { for exponential windowing. }\end{cases}$

We define the procedures for updating, downdating, and deflating the decomposition.

\section{Update_URV:}

1) If a row $y^{H}$ is to be deleted from $A$, downdate the factorization.

2) If exponential windowing is used, multiply $R$ by the forgetting factor.

3) Transform coordinates in the incoming row by multiplying by $V: z^{H}=z^{H} V$. 


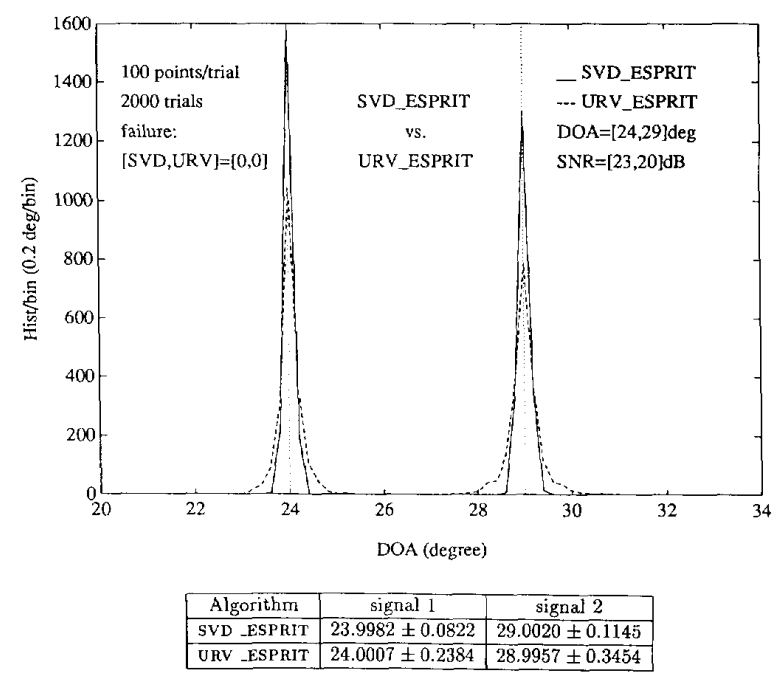

Fig. 1. Histogram and estimated means and variances from ESPRIT (SVD) and ESPRIT (URV) for fixed sources.

4) Let $\hat{z}$ denote the vector formed by deleting the first $d$ components of $z$.

5) If $\sqrt{\|G\|^{2}+\|\hat{z}\|^{2}}>$ tol_u, then there is a potential increase in rank:

Tentatively increase the rank estimate: $d=d+1$. Compute rotations to reduce $\hat{z}$ to a multiple of the first unit vector. Apply these rotations to $z^{H}$ from the right and to $V^{\mathrm{H}}$ from the left. Apply them as right rotations to $R$, interleaved with a set of left rotations to maintain triangular form. end_if

6) Compute and apply left rotations to reduce the matrix $\left(\begin{array}{c}R \\ z^{H}\end{array}\right)$ to triangular form by zeroing the elements of $z$. 7) Deflate.

DEFLATE

while $d>0$,

1) Perform a condition number estimation on the leading principal submatrix of $R$ of dimension $d$, to compute an approximate null vector $w$.

2) Compute rotations to reduce $w$ to a multiple of the last unit vector. Apply these rotations to $R$ from the right and to $V$ from the left. Let $\hat{r}$ denote the element of $R$ in position $(d, d)$.

3) If $\sqrt{\|G\|^{2}+|\hat{r}|^{2}}<$ tol_d, then

$d=d-1$

Perform a polishing step:

Compute right rotations to reduce the $d+1$ st column of $R$ to 0 in rows 1 to $d$. Apply these rotations to $R$ from the right and to $V$ from the left.
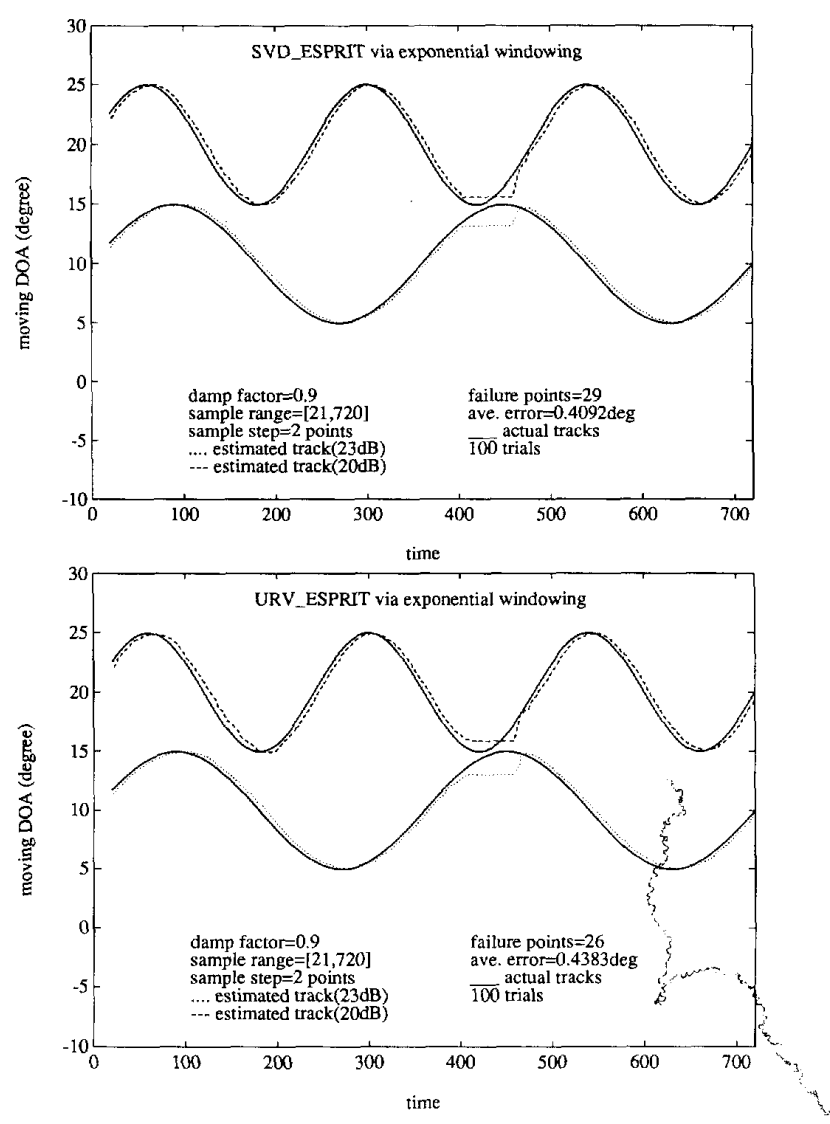

Fig. 2. Estimated time-varying DOA's for close sources using exponential windowing.

Apply left rotations to $R$ to reduce the $d$ row of $R$ to 0 in columns 1 to $d$.

else

break

end_if

end_while

\section{DOWNDATE:}

1) Following Chambers' algorithm, compute a sequence of left rotations to transform the matrix

$$
\left(\begin{array}{c}
R \\
0^{T}
\end{array}\right)
$$

to the matrix

$$
\left(\begin{array}{c}
\hat{R} \\
y^{\mathrm{H}} V
\end{array}\right)
$$

2) Deflate.

\section{EXPERIMENTAL RESULTS}

In this section, we present some simulation results that compare the performance of the two algorithms: SVD ESPRIT (using the estimated covariance matrix) and URV ESPRIT. 

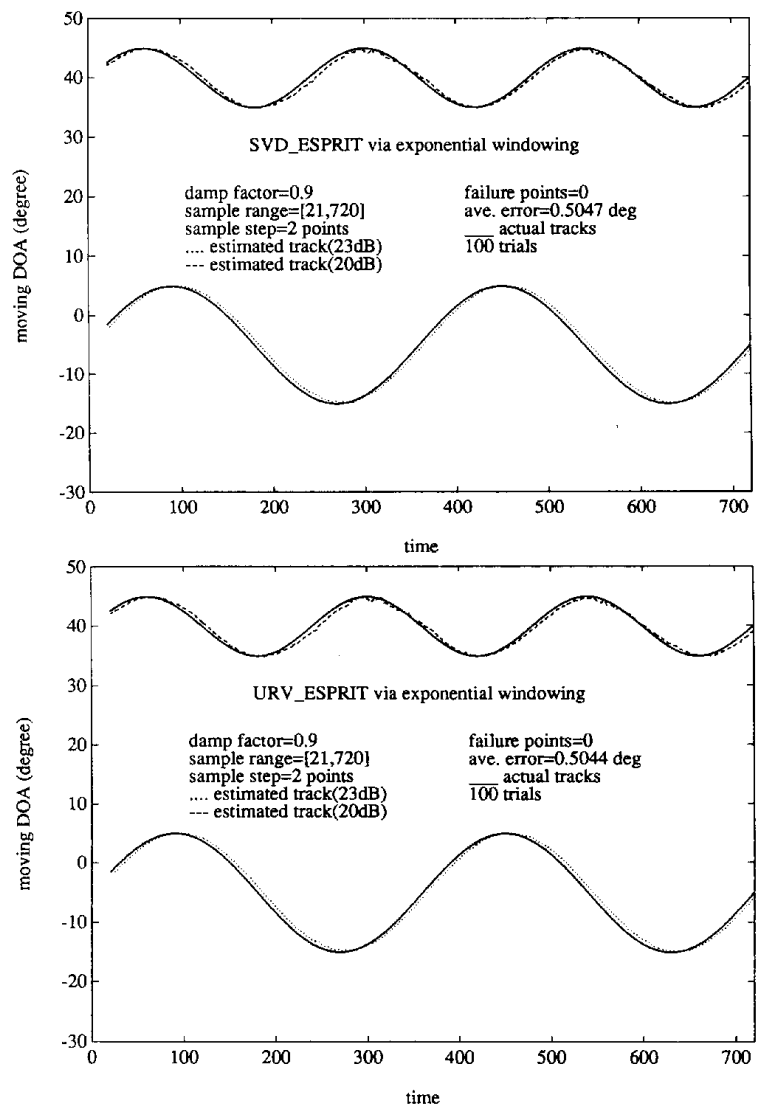

Fig. 3. Estimated time-varying DOA's for well-separated sources using exponential windowing.

We use a five-pair ( $m=10$ ) linear array with pair spacing $\lambda / 4$. The pairs are equally spaced on a line with relative locations $[0,1,2,3,4] \lambda$. The two signals are narrow-band with signal-to-noise ratio (SNR) $23 \mathrm{~dB}$ and $20 \mathrm{~dB}$, respectively. The noise is white Gaussian, and the algorithms were tested with duplicate data samples in order to make a fair comparison.

We say that an algorithm failed at a particular time if it estimated more or fewer than two signals.

The first example concerns two fixed signal sources located at $24^{\circ}$ and $29^{\circ}$. For each trial, we estimated the DOA's based on 100 randomly generated data samples for each signal, with $50 \%$ correlation between the two sets of samples. We ran 2000 trials. Fig. 1 shows a histogram and tabular summary of the results. Both algorithms were quite successful. This shows that we are not sacrificing much accuracy by substituting the more economical URV for the SVD..

Other experiments concerned time-varying DOA's. We used exponential windowing, with a forgetting factor $\mu=.9$. Two data sets were used, one involving close sources located at

$$
\begin{aligned}
& 10^{\circ}+5^{\circ} \sin (2 \pi n / 360) \\
& 20^{\circ}+5^{\circ} \sin (2 \pi n / 240)
\end{aligned} \quad n=1,2, \ldots, 719
$$
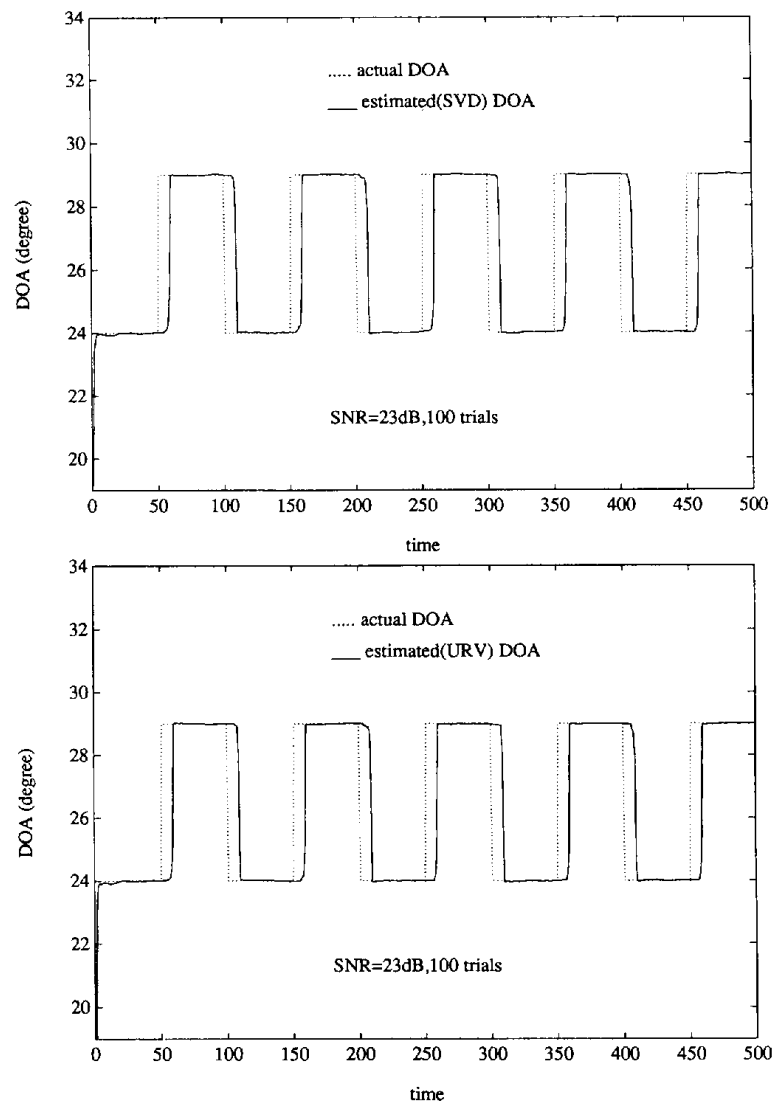

Fig. 4. Estimated time-varying DOA's for instantaneously changing signals using rectangular windowing.

and one involving well-separated sources

$$
\begin{aligned}
& -5^{\circ}+10^{\circ} \sin (2 \pi n / 360) \\
& 40^{\circ}+5^{\circ} \sin (2 \pi n / 240)
\end{aligned} \quad n=1,2, \ldots, 719 .
$$

This corresponds to a sampling rate of 1 data point per $.11^{\circ}$, $.08^{\circ}$, or $.05^{\circ}$ change in angle. Typical radar applications produce 1 point per $10^{(10-5) \circ}$ change, so our experimental setup is much more demanding.

Experiments varying the rank determination tolerances $\psi_{d} \in$ $\{3,6\}$ and $\psi_{u} \in\{0,1,2\}$ for signals of different signal-tonoise ratio, separation angle, and rate of change of DOA showed that results improved as $\psi_{u}$ was decreased, but that the value of $\psi_{d}$ is more problem dependent. The error model we use assumes known variance accounting for random errors in the measurements, but not for movement of the sources. As the rate of change in the DOA increases, the value of $\psi_{d}$ must be increased in order to account for this extra source of error. The experiments presented here use the values $\psi_{d}=3$ and $\psi_{u}=1$.

Fig. 2 gives the results for the close sources, and Fig. 3 gives the results for the well-separated sources. The DOA's were updated every two data samples. Both algorithms perform 

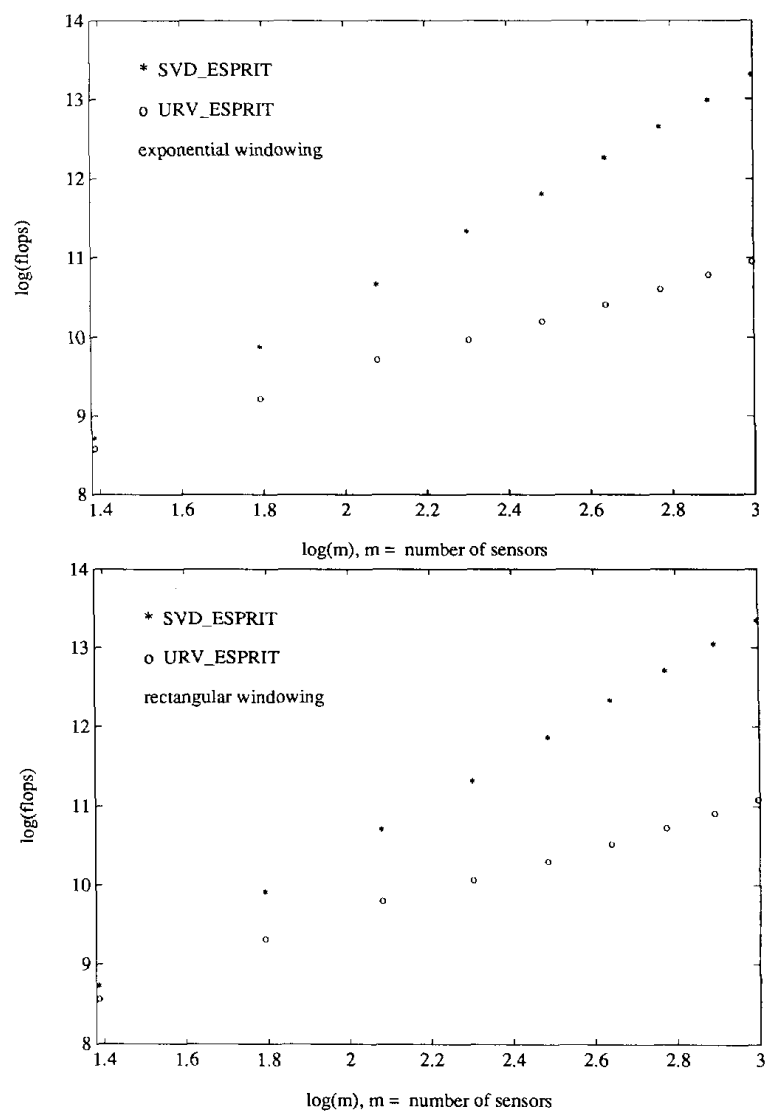

Fig. 5. Number of floating point operations for processing a new data point for $m=4, \ldots, 20$ sensors.

quite well, and give similar numbers of failures. This gives us confidence that the two algorithms would have similar failure behavior even if the rank determination procedure was changed. Average error was also similar for both algorithms. There seems to be no reason to prefer the more expensive SVD-based algorithm over the URV, and the results indicate that, in practice, low sampling rates can be tolerated well. Extra data could be used for noise reduction through averaging over short time periods, feeding the time-averaged data to ESPRIT. Errors in both algorithms increase as the sources converge, with breakdown at approximately the same point.

As a final example, to demonstrate tracking of instantaneously changing signals, we assumed that there were two signal sources located at $24^{\circ}$ and $29^{\circ}$, each with SNR $23 \mathrm{~dB}$, and that the signals alternatively appear and disappear. We took a rectangular window of size 10. Fig. 4 shows the similar good performance of the two algorithms.

We computed the costs required for both algorithms. We recorded the number of floating point operations needed for the well-separated sources problems with various number of sensors $m=4,6, \ldots, 20$. Fig. 5 gives the $\log \log$ plot of the average number of floating point operations required for processing one new data point for exponential and rectangular windowing. The slopes show that, as predicted, the costs of the SVD-based algorithm increase much faster than the URV.

These experimental results lead us to believe that the URVbased ESPRIT algorithm can be successfully used for real-time tracking of time-varying signals.

\section{SUMMARY}

We have presented a new variant of the ESPRIT algorithm that has significant computational advantages over previous ones and that allows ESPRIT now to be used for realtime signal processing involving moving sources. It has the following features:

- The storage requirement is $O\left(m^{2}\right)$ (plus $m n$ for rectangular windowing).

- The work per update is $O\left(m^{2}+d^{3}\right)$.

- Its performance is similar to SVD-based algorithms, at greatly reduced computational cost, and it admits an efficient parallel realization.

We have explained the relation between the SVD and URV versions of the algorithm and the accuracy of the subspace determination. We also presented a downdating algorithm for URV, along with partial results on the stability of sequences of updates and downdates. We showed that the AIC and MDL schemes can also be used with the URV to estimate the number of signals, although further research is needed into rank determination for systems with moving sources.

\section{ACKNOWLEDGMENT}

The comments of D. Fuhrman and the referees resulted in significant changes to this presentation.

\section{REFERENCES}

[1] R. Roy and T. Kailath, "ESPRIT-Estimation of signal parameters via rotational invariance techniques," in Signal Processing Part II: Control Theory and Applications, F. A. Grünbaum, J. W. Helton, and P. Khargonekar, Eds. New York: Springer-Verlag, pp. 369-411, 1990.

[2] R. O. Schmidt, "A signal subspace approach to multiple emitter location and spectral estimation," Ph.D. thesis, Stanford University, 1981.

[3] W. Ferzali and J. Proakis, "Adaptive SVD algorithm with application to narrowband signal tracking, in SVD and Signal Processing, II, R. J. Vaccaro, Ed. Amsterdam: Elsevier Science Publishers, 1990, pp. $149-160$.

4] M. Moonen, P. Van Dooren, and J. Vandewalle, "Combined Jacobi-type algorithms in signal processing," in SVD and Signal Processing, $I, \mathrm{R} . \mathrm{J}$. Vaccaro, Ed. Amsterdam: Elsevier Science Publishers, pp. 177-188.

[5] G. W. Stewart, "An updating algorithm for subspace tracking," IEEE Trans. Signal Processing, vol, 40, pp. 1535-1541, 1992.

[6] M. F. Griffin, E. C. Boman, and G. W. Stewart, "Min-norm updating with the rank-revealing URV decomposition," in Proc. ICASSP-92 (San Francisco), 1992.

[7] J. R. Bunch and C. P. Nielsen, "Updating the singular value decomposition," Numerische Mathematik, vol. 31, pp. 111-129, 1978.

[8] R. Mathias and G. W. Stewart, "A block QR algorithm and the singular value decomposition," Linear Algebra and Its Applications, vol. 182, pp. 91-100, 1993.

[9] G. H. Golub and C. F. Van Loan, Matrix Computations. Baltimore: Johns Hopkins Univ. Press, 2nd ed., 1989.

[10] N. J. Higham, "A survey of condition number estimation for triangular matrices," SIAM Review, vol. 29, pp. 575-596, 1987.

[11] M. Moonen, "Jacobi-type updating algorithms for signal processing, systems identification and control," Ph.D. thesis, Katholieke Universiteit Leuven, Belgium, 1990.

[12] G. W. Stewart, "Error analysis of QR updating with exponential windowing," Math. Computat., vol. 59, pp. 135-140, 1992. 
[13] R. D. DeGroat and R. A. Roberts, "Efficient numerically stabilized rank-one eigenstructure updating," IEEE Trans. Acoust., Speech, Signal Processing, vol. 38, pp. 301-316, 1990.

[14] M. Moonen, P. Van Dooren, and J. Vandewalle. "A note on 'Efficient numerically stabilized rank-one eigenstructure updating," IEEE Trans. Signal Processing, vol. 39, pp. 1911-1913, 1991. Reply by DeGroat, pp. 1913-1914.

115] A. Edelman and G. W. Stewart, "Scaling for orthogonality," IEEE Trans. Signal Processing, vol. 41, pp. 1676-1677, 1993.

[16] J. J. Dongarra et al., LINPACK User's Guide. Philadelphia: SIAM, 1979.

[17] J. M. Chambers, "Regression updating," J. Amer. Stat. Assn., vol. 66 pp. $744-748,1971$

[18] G. W. Stewart, "On Chambers' downdating algorithm and the stability of sequential updates and downdates," Tech. Rpt., Inst. for Adv. Comput. Studies and Comput. Sci. Dept., Univ, of Maryland, College Park, to appear.

[19] S. Haykin, Adaptive Filter Theory. Englewood Cliffs, NJ: Prentice Hall, 1991 .

[20] Q. Wu and D. Fuhrmann, "A parametric method for determining the number of signals in narrow-band direction finding," IEEE Trans. Signal Processing, vol. 39, pp. 1848-1857, 1991.

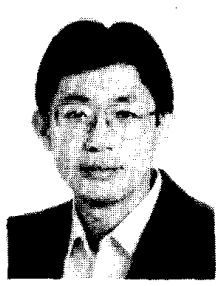

K. J. Ray Liu (S'86-M'90-SM'93) received the B.S. degree in electrical engineering from National Taiwan University in 1983, the M.S.E. degree in electrical engineering and computer science from the University of Michigan, Ann Arbor, in 1987 and the Ph.D. degree in electrical engineering from the University of California, Los Angeles, in 1990.

During 1983-1985 he served in the Signal Corps,

Taiwan, as a Communications Officer. $\mathrm{He}$ then became a Teaching and Research Assistant at the University of Michigan and the University of California, Los Angeles. Since 1990, he has been an Assistant Professor in the Electrical Engineering Department and Institute for Systems Research, University of Maryland, College Park. His research interests span all aspects of high performance computational signal processing, including parallel and distributed processing, fast algorithms, VLSI, and concurrent architecture, with application to image/video, radar/sonar, communications, and medical and biomedical technology.

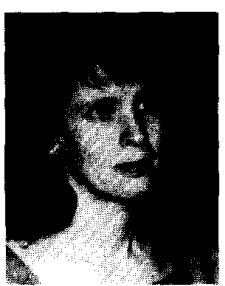

Dianne P. O'Leary received the B.S. degree in mathematics from Purdue University in 1972, and the Ph.D. in computer science from Stanford University in 1976

She is a Professor in the Department of Computer Science at the University of Maryland. Her research interests are in parallel numeric algorithms, digital signal processing, solution of ill-posed problems, and computational linear algebra and optimization.

Dr. O'Leary is a member of the editorial boards of IEEE Computational Science and Engineering and SIAM Review.



G. W. Stewart received a degree in mathematics in 1969 under the late Alston Householder.

$\mathrm{He}$ is a Professor in the Computer Science Department and Research Professor in the Institute for Advanced Computer Studies at the University of Maryland, College Park. He is the author of many papers on various aspects of numerical analysis and matrix computation, with applications in statistical computing, signal processing, and stochastic processes. His books include Introduction to Matrix Computation and, with J. G. Sun, Matrix Perturbation Theory. He is a coauthor of the LINPACK package for linear algebra.

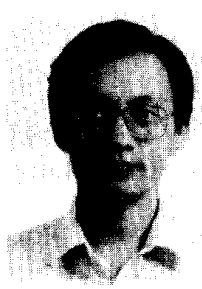

Yuan-Jye J. Wu received the B.S. degree in applied mathematics from National Chung-Hsing University, Taichung, Taiwan, in 1986. He is a Ph.D. candidate in applied mathematics at the University of Maryland, College Park.

He is currently a Research Assistant in the Department of Computer Science, His research interests include digital signal processing, numerical linear algebra, and Markov chains. 